\title{
Capillaria hepatica in Rattus Spp. Captured in Sanandaj and Orally uninfected Balb/C Mice With Embryonated Eggs
}

\author{
Ghasem Zamini', Mohammad Bagher Khadem-Erfan ${ }^{1,2}$, Ashkan Faridi1,2* \\ 'Department of Parasitology and Mycology, Faculty of Medicine, Kurdistan University of Medical Sciences, Sanandaj, Iran \\ ${ }^{2}$ Cellular and Molecular Research Center, Kurdistan University of Medical Sciences, Sanandaj, Iran
}

\author{
*Correspondence to \\ Ashkan Faridi, \\ Tel: +989188792100 \\ Email: ashkan.faridi@yahoo.com
}

Received April 16, 2017 Accepted August 20, 2017 Published online September 31, 2017

\begin{abstract}
Introduction: Calodium hepaticum (syn. Capillaria hepatica) is a nematode with worldwide distribution among rodents (Rattus rattus, Rattus norvegicus) which can also infect human beings through ingestion of embryonated eggs and cause the zoonotic disease of capillariasis philippinensis. This study aimed to determine the C. hepatica infection in Rattus spp. in Sanandaj, Kurdistan province of Iran, as well as orally infecting of Balb/c mice with embryonated eggs. Methods: Area of study was the city of Sanandaj located in the west of Iran, wherein $4 R$. norvegicus, 6 R. rattus and 10 Mus musculus were captured using live traps in a period of more than 2 years. Livers were examined for $C$. hepatica infection, sectioned, and then stained with hematoxylin and eosin. Embryonated eggs were used in order to experimentally infect $3 \mathrm{Balb} / \mathrm{c}$ mice orally.

Results: Capillaria hepatica was detected in 5 out of 20 livers (25\%); rates of infection were $50 \%$ (2/4) for $R$. norvegicus, $50 \%$ (3/6) for $R$. rattus, and $0 \%(0 / 10)$ for $M$. musculus. Cross sections of the livers showed active infections as eggs were deposited beside the worms, and they were also observed inside the female worms. After performing the autopsy on 3 mice that had been infected experimentally, there was no sign of infection based on gross and microscopic examinations. Conclusion: This study was the first report on C. hepatica infection in Rattus spp. in Sanandaj. The main reservoir hosts of $C$. hepatica infection in different areas of Sanandaj were $R$. rattus and R. norvegicus. On the other hand, with regard to limited sample size of study, no significant role could be considered for either infection or transmission of $C$. hepatica for M. musculus in these areas.

Keywords: Parasitic infection, Calodium hepaticum, Capillaria hepatica, Liver, Rodent
\end{abstract}

\section{Introduction}

Capillaria hepatica (syn. Calodium hepaticum) is a nematode, first described by Bancroft in 1893. The parasite belongs to the order of Trichinellida and Capillariidae. ${ }^{1}$ It is distributed worldwide among rodents, and also has been found in more than 140 mammalian species such as human beings, which causes the zoonotic disease of capillariasis. $^{2}$ The size of an adult parasite is ranged from 30 to $50 \mathrm{~mm}$ in length in males and females. ${ }^{1}$ The eggs are bi-operculatestructured with a tray-like shape, and have a pitted shell, and the apical plugs do not protrude. ${ }^{3}$ Eggs are embryonated within 5 to 7 weeks outside the host and transmitted directly through being ingested by the soil or animal carcasses without the need for an intermediate host. After ingestion of infective eggs, L1 larvae migrate to the cecum, penetrate into the intestinal walls, enter the bloodstream, and finally reach the hepatic parenchyma, where they turn from L2 into L4 larvae, and then into adult worms in about 1 month. The mature adult worms mate and release the cluster of fertilized eggs around the female releases. The female dies after a period of approximately 30 days after laying eggs, and immature fertilized eggs remain viable up to 120 days

Copyright (C) 2017 The Author(s); Published by Zabol University of Medical Sciences. This is an open-access article distributed under the terms of the Creative Commons Attribution License (http://creativecommons.org/licenses/by/4.0), which permits unrestricted use, distribution, and reproduction in any medium, provided the original work is properly cited. 
in host's liver. ${ }^{4}$ In 1924, the first human infection was reported by McArthur, and after that, this infection was reported occasionally from different parts of the world. ${ }^{5}$ Two infection types caused by $C$. hepatica are: spurious infection when host ingests unembryonated eggs, and true hepatic infection following the ingestion of embryonated eggs. ${ }^{6}$ Site of true infection is liver parenchyma, where in case of human infection, symptoms such as prolonged fever, splenomegaly, acute liver inflammation, peritonitis, abdominal pain, ascites and eosinophilia may occur, and occasionally cause severe and fatal infections. ${ }^{7,8}$

Rodents are the largest mammalian group on the earth. ${ }^{9}$ Synanthropic rodents that live in close contact with human habitats in urban areas worldwide are house mouse (M. musculus), black rat ( $R$. rattus), and brown rat $(R$. norvegicus). ${ }^{10}$ One of the critical public health problems to human populations are Rattus spp. which harbor endoparasite nematodes such as Capillaria hepatica, Aspiculuris tetraptera, Syphacia obvelata, Trichinella spp., Stronglyloid muris, Trichosomoides crassicauda; trematodes such as Schistosoma spp.; cestodes such as Hymenolepis nana, Hymenolepis diminuta, Taenia taeniaeformis; acanthocephalans such as Moniliformis moniliformis; protozoa such as Cryptosporidium spp., Babesia spp., Trypanosoma spp., Leishmania spp., Trichomonas muris, Giardia spp, Sarcocystis spp., ${ }^{11-14}$ and many other parasites, bacteria and viruses that most of them could cause zoonotic diseases in humans. ${ }^{15}$

Rodents, especially the genus Rattus, are considered to be the main reservoir and primary host of $C$. hepatica ${ }^{15}$ in which prevalence rate of infection can reach to $100 \%$ in some areas, and this infection has been reported from many parts of the world ${ }^{16}$. Active life cycle of the parasite in different geographical locations can be a potential risk for the public health ${ }^{17}$; so this study aimed to determine the C. hepatica infection in Rattus spp. in Sanandaj, Kurdistan province of Iran, and investigate the sensitivity of laboratory mice to the maintenance of the parasite in vivo with embryonated eggs orally.

\section{Methods}

Area of Study

Sanandaj is the capital of Kurdistan province located at $35^{\circ} 18^{\prime} 52^{\prime \prime} \mathrm{N}, 46^{\circ} 59^{\prime} 32^{\prime \prime} \mathrm{E}$, with the elevation of 1521 $\mathrm{m}$ above the sea level, in the west of Iran. Map of the study area was drawn by Arc GIS version 10 (Figure 1C). The population of this city in 2014 was estimated to be 432330. Twenty rodents were collected from different urban areas of Sanandaj in a period of more than 2 years.

Rodent Collection, Identification And Separation of Capillaria hepatica From Infected Liver

Four R. norvegicus, 6 R. rattus, and 10 M. musculus from the study area were captured using live trap each time in interval of 1 day between 2 weeks. The mice were labeled and transferred to the laboratory of parasitology at the Kurdistan University of Medical Sciences, then sacrificed humanely by an overdose of ketamine and xylazine intraperitoneally injected, and identified based on diagnostic keys with regard to the proportion of the size of tail to whole body, ear to eye distance and dental pattern. ${ }^{18}$ After autopsy, we speculated whether the livers were infected with $C$. hepatica. Worms that had infected the livers were separated from the liver parenchyma using fine needles under stereomicroscope. Then, all the livers were separately homogenized, washed with PBS, and centrifuged at $1000 \mathrm{~g}$ at least three times, and obtained sediments were observed by a microscope.

\section{Sectioning and Staining}

One of the infected livers was processed and stained with hematoxylin and eosin based on a prior study ${ }^{19,20}$. Briefly, one part of the liver was trimmed, fixed in $10 \%$ formaldehyde for 24 hours, dehydrated in each of the $50 \%, 70 \%, 90 \%$ and 2 series of $100 \%$ ethanol for 15 minutes. Then, clearing was done with 2 series of xylene for 20 minutes. Hot paraffin $\left(50^{\circ} \mathrm{C}\right)$ was added to infiltrate the tissue for a sequence of two 30-minute periods and it was embedded carefully in paraffin cassettes to determine the plane of section. Sectioning was done with microtome in 2-4 mm thickness, and the sections were mounted on microscope slides. Then, they were stained with hematoxylin and eosin and finally the sections were examined under a light microscope.

Embryonation of the Eggs of Capillaria hepatica

Two sets of the eggs that were derived from infected livers were cultivated at first in potassium dichromate $2.5 \%$ and then in distilled water with $2 \%$ formaldehyde at room temperature until the eggs were embryonated.

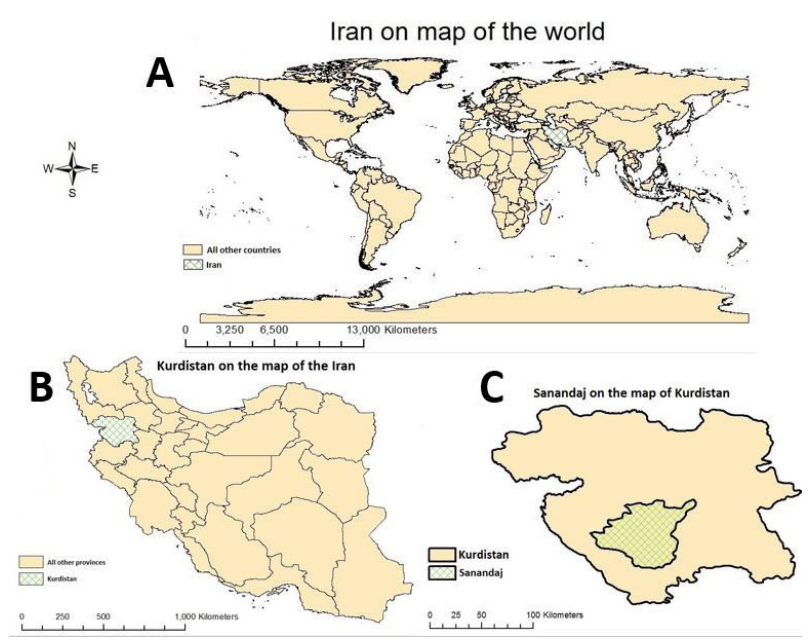

Figure 1. Map of Sanandaj, west of Iran, area from where the rodents were collected. (A) Iran on the map of the world, (B) Kurdistan on the map of Iran, (C) Study area of Sanandaj on the map of Kurdistan, drawn by Arc GIS version 10 . 
Experimental Infection of Balb/c Mice With Capillaria hepatica

After observing motile larvae within the eggs, at least 30 eggs with motile larvae from the existing mix of eggs from cultivation in $2.5 \%$ potassium dichromate, distilled water and $2 \%$ formaldehyde were washed with normal saline and centrifuged at $500 \mathrm{~g}$ for 3 times. Then, they were administered orally to 3 six-week-old male $\mathrm{Balb} / \mathrm{c}$ mice using gavage needles number 18. The first mouse was sacrificed 1 month after administration, the second was sacrificed 3 months after administration, and the third was sacrificed 9 months after administration of the eggs ${ }^{21}$

\section{Results}

Overall, 20 samples including $4 R$. norvegicus, 6 R. rattus (Figure 2A) and $10 \mathrm{M}$. musculus were collected. C. hepatica was detected in 5 out of 20 livers (25\%) (Figure 2B), and worms (Figure 2C) and eggs (Figure 2D) were separated from the livers. The liver infection was $50 \%(2 / 4)$ in $R$. norvegicus, $50 \%(3 / 6)$ in $R$. rattus, and $0 \%(0 / 10)$ in $M$. musculus.
Cross section showed active infection of the liver with eggs deposited beside the worm (Figure 3A) and even inside the female worms (Figure 3B). Furthermore, in another part of the same liver, a fibrous capsule was observed around unembryonated eggs that could be related to a prior infection with $C$. hepatica (Figure 3C). Due to hyper infection, the lesions were observed in different parts of liver lobes and in some cases the worms could not be separated intact from the liver.

Three days after oral administration of the eggs with motile larvae (Figure 2E), the mice were reluctant to move and were hunched, and their fur were ruffled. After autopsy, by gross and microscopy examination, signs of infection were observed in none of the infected Balb/c mice.

\section{Discussion}

This study was the first report on $C$. hepatica infection in Rattus spp. in Sanandaj, capital city of Kurdistan province, in the west of Iran. Due to the zoonotic importance of capillariasis and its potential in infecting humans, for

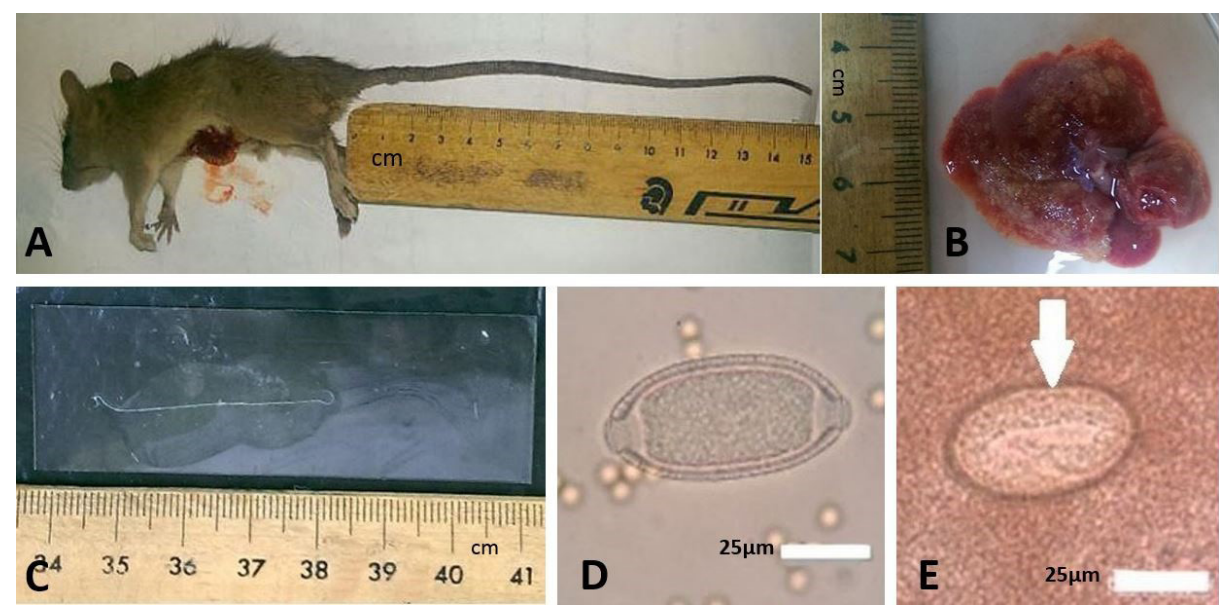

Figure 2. Rattus rattus With Capillaria hepatica Infection Captured in Sanandaj. (A) R. rattus captured in Sanandaj with macroscopic liver lesion, (B) Abnormal liver with rough different colored spots on the surface of the liver, (C) The adult worm of $C$. hepatica isolated from an infected liver was measured approximately $35 \mathrm{~mm}$, (D) Brown oval shaped egg of $C$. hepatica with the size of approximately 55 microns and shallow polar knobs at both ends and double layered shell with minipores that can be seen in the outer shell of C. hepatica, (E) Motile larvae within the egg of $C$. hepatica after 39 days of cultivation in potassium dichromate $2.5 \%$ at room temperature.
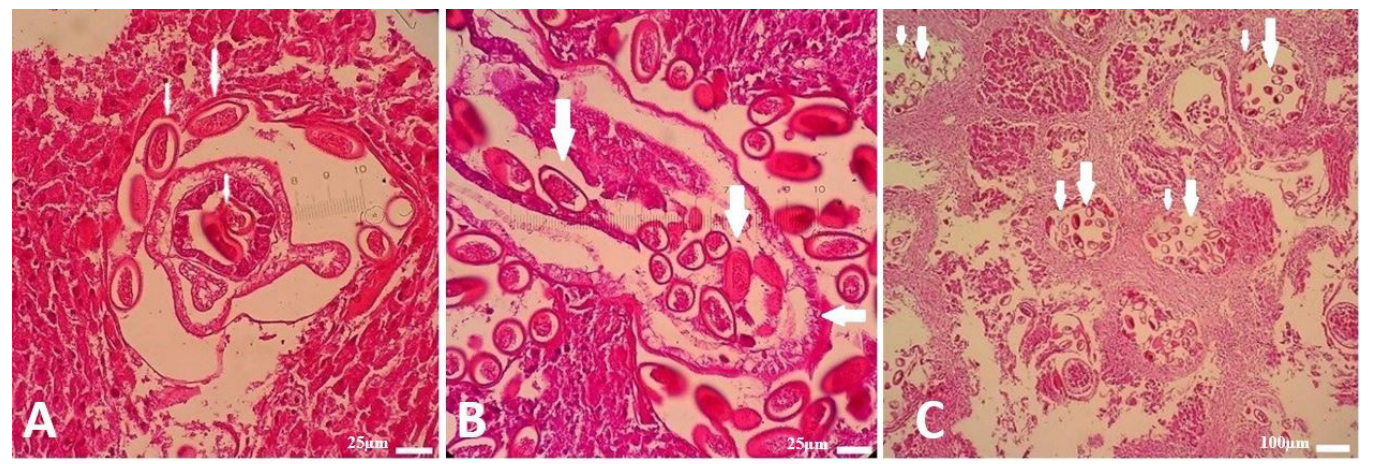

Figure 3. Hepatic Tissue of Rattus rattus Infected With $C$. hepatica. H \& E staining. (A) Eggs deposited beside the female worm and inside the worm, (B) Cross section of a worm inside liver parenchyma where eggs are inside the female worm. (C) Cluster of bioperculate eggs and fibrous tissue around the eggs. 
example the true case report of a severe Capillaria infection in a 5-year-old child of Iran in $2015,{ }^{7}$ and the fact that genus Rattus is considered a reservoir host for this infection, infected rodents are reported as risk factors for public health. Rodents' infection has been reported from different parts of Iran including Kermanshah in $23 R$. norvegicus and $5 R$. rattus with the infection rate of $13.04 \%$ and $20.0 \%$, respectively. ${ }^{22}$ The infection was reported in Ardabil in 120 Meriones persicus with the infection rate of $6.9 \%{ }^{23}$.

The infection rates in some other parts of the world were $36 \%$ in captured $R$. norvegicus in Milan, Italy ${ }^{24}, 8 \%$ in 3 noncommensal rodent species from Geneva, Switzerland, ${ }^{25}$ $87.9 \%$ in Norway rats in Baltimore, Maryland, USA, ${ }^{11}$ and $35 \%$ of $R$. rattus and $54 \%$ of $R$. norvegicus in Australia ${ }^{26}$

However in several studies in Iran, the researchers were not able to find Capillaria infection in their studies, for example in a study performed in Ahvaz, ${ }^{27}$ as well as in another study conducted in Dezful and Andimeshk, in Khuzestan province ${ }^{28}$ and also in wild rodents in Sistan and Baluchistan province. ${ }^{29}$ This probably means that the endemic area of $C$. hepatica is confined to specific parts of Iran and is not distributed uniformly in all parts of the country. In order to determine the real status of these parasites, conducting more studies in other parts of Iran is required.

The rats captured for the study of Capillaria infection were infected similar to studies reported in other parts of the world, including Milan, Italy, ${ }^{24}$ Baltimore, Maryland, ${ }^{30}$ Brazil, ${ }^{31}$ Switzerland, ${ }^{25}$ and Egypt. ${ }^{32}$ In addition, the rats captured from Sanandaj showed partial resistance to this nematode since they were heavily infected, without showing apparent clinical signs and had fibrous clusters of eggs in one part of their liver as well as active infection of live adult worms in other parts (Figure 3C). It can be concluded that the rats of these areas, in addition to being reservoir hosts for $C$. hepatica nematode, are also a potential risk factor to the inhabitants of Sanandaj. However, 10 house mice (M. musculus) captured from urban and suburban areas of Sanandaj, showed no evidence of Capillaria infection in their livers, and surprisingly we failed to infect Balb/c mice with embryonated eggs orally. Although some studies have shown C. hepatica in the liver of house mice and even named this nematode as a potential organism for biological control of mice plague through falling natality as well as decreased survival rate of mice from lactation period. ${ }^{33,34}$ In addition, another study showed Capillaria in the liver of house mice and considered house mice as a source of infection, similar to Rattus species ${ }^{35}$ Though in some studies with regard to low prevalence of Capillaria spp. in M. musculus, it has been suggested that this species is less affected by $C$. hepatica and house mice, also, is rarely infected by it. ${ }^{36}$ Studies carried out on M. musculus showed differences in the susceptibility of this species to Capillaria infection.
Findings of this study showed no infection with Capillaria spp. in house mice, which could mean house mice probably do not play an active role in the life cycle of this disease in Sanandaj.

The possible mechanism for the presence of infection in rats and no infection in house mice in this study might be related to various forms of behavioral patterns in these species, and conditions that separate community organization and population dynamics in these 3 species; so maybe none of them enters the territory of other species. ${ }^{37}$ Another possible mechanism might be related to the genetic diversity of $C$. hepatica in different geographical regions, though proving this theory requires conducting molecular studies in this area.

\section{Conclusion}

The main reservoir hosts of $C$. hepatica in Sanandaj are $R$. rattus and $R$. norvegicus, and they could be considered as risk factors for public health. On the other hand, $M$. musculus that was included in this study was not infected experimentally with Capillaria, which probably means that no significant role for the infection or transmission of Capillaria could be considered for it; and unsuccessful attempts to infect $\mathrm{Balb} / \mathrm{c}$ mice experimentally, with embryonated eggs orally, could comply with this claim, or at least should be carried out to validate this finding. Lastly, due to the high prevalence rate of $C$. hepatica in rodents of the study area, obscured liver syndromes particularly in children should be considered as suspicious cases of Capillaria infection by healthcare providers, and it is recommended investigating them via various methods, such as serological tests, biopsy and magnetic resonance imaging (MRI).

\section{Ethical Approval}

The study was supervised and approved by Ethics Committee of Kurdistan University of Medical Sciences for handling and anesthesia of animals sacrificed in the study.

\section{Competing Interests}

Authors declare that they have no competing interests.

\section{Acknowledgment}

The authors' special thanks go to Babak Dadizadeh for proofreading of manuscript.

\section{Reference}

1. Wright KA. Observations on the life cycle of Capillaria hepatica (Bancroft, 1893) with a description of the adult. Can J Zool. 1961;39(2):167-182. doi:10.1139/z61-022.

2. Fuehrer HP. An overview of the host spectrum and distribution of Calodium hepaticum (syn. Capillaria hepatica): part 1-Muroidea. Parasitol Res. 2014;113(2):619640. doi:10.1007/s00436-013-3691-x. 
3. Fugassa MH, Taglioretti V, Goncalves ML, Araujo A, Sardella NH, Denegri GM. Capillaria spp. eggs in Patagonian archaeological sites: statistical analysis of morphometric data. Mem Inst Oswaldo Cruz. 2008;103(1):104-105.

4. Klisiowicz Ddo R, Reifur L, Shimada MK, Haidamak J, Cognialli RC, Ferreira T. High occurrence of Calodium hepaticum (syn. Capillaria hepatica) spurious infection in a village in the Atlantic Forest of southern Brazil. Mem Inst Oswaldo Cruz. 2014;109(3):371-373.

5. Fuehrer HP, Igel P, Auer H. Capillaria hepatica in man--an overview of hepatic capillariosis and spurious infections. Parasitol Res. 2011;109(4):969-979. doi:10.1007/s00436011-2494-1.

6. Fan PC, Chung WC, Chen ER. Capillaria hepatica: a spurious case with a brief review. Kaohsiung J Med Sci. 2000;16(7):360-367.

7. Aghdam MK, Karimi A, Amanati A, et al. Capillaria hepatica, A case report and review of the literatures. Arch Pediatr Infect Dis. 2015;3(2):e19398. doi:10.5812/ pedinfect.19398.

8. Juncker-Voss M, Prosl H, Lussy H, Enzenberg U, Auer H, Nowotny N. Serological detection of Capillaria hepatica by indirect immunofluorescence assay. J Clin Microbiol. 2000;38(1):431-433.

9. Singla N, Dhar P, Singla LD, Gupta K. Patho-physiological observations in natural concurrent infections of helminth parasites of zoonotic importance in the wild rodents, Bandicota bengalensis. J Parasit Dis. 2016;40(4):14351442. doi:10.1007/s12639-015-0709-6.

10. Meyer A. Urban commensal rodent control: Fact or fiction? In: Singleton GR, Krebs CJ, Spratt DM, eds. Rats, Mice and People: Rodent Biology and Management. Canberra: Australian Centre for International Agricultural Research; 2003:446-450.

11. Easterbrook JD, Kaplan JB, Vanasco NB, et al. A survey of zoonotic pathogens carried by Norway rats in Baltimore, Maryland, USA. Epidemiol Infect. 2007;135(7):1192-1199. doi:10.1017/s0950268806007746.

12. Ogunniyi T, Balogun H, Shasanya B. Ectoparasites and endoparasites of peridomestic house-rats in ile-ife, Nigeria and implication on human health. Iran J Parasitol. 2014;9(1):134-140.

13. Priscilla D, Jambari HA, Meenakshii N. Prevalence of mouse and rat parasites in resource recovery plants, farms and housing areas of Southern Selangor: implication for public health. Pertanika J Trop Agric Sci. 2015;38(3):309320.

14. Tung KC, Hsiao FC, Wang KS, Yang CH, Lai CH. Study of the endoparasitic fauna of commensal rats and shrews caught in traditional wet markets in Taichung City, Taiwan. J Microbiol Immunol Infect. 2013;46(2):85-88. doi:10.1016/j.jmii.2012.01.012.

15. Himsworth CG, Parsons KL, Jardine C, Patrick DM. Rats, cities, people, and pathogens: a systematic review and narrative synthesis of literature regarding the ecology of rat-associated zoonoses in urban centers. Vector Borne Zoonotic Dis. 2013;13(6):349-359. doi:10.1089/ vbz.2012.1195.

16. Rothenburger JL, Himsworth CG, Chang V, LeJeune
M, Leighton FA. Capillaria hepatica in wild Norway rats (Rattus norvegicus) from Vancouver, Canada. J Wildl Dis. 2014;50(3):628-633. doi:10.7589/2013-09-256.

17. Battersby SA, Parsons R, Webster JP. Urban rat infestations and the risk to public health. J Environ Heal Res. 2002;1(2):57-65.

18. Stuart C. Field guide to Mammals of Southern Africa. Cape Town, South Africa: Struik; 2001.

19. Fischer AH, Jacobson KA, Rose J, Zeller R. Hematoxylin and eosin staining of tissue and cell sections. CSH Protoc. 2008;2008:pdb.prot4986. doi:10.1101/pdb.prot4986

20. Hewitt SM, Lewis FA, Cao Y, et al. Tissue handling and specimen preparation in surgical pathology: issues concerning the recovery of nucleic acids from formalinfixed, paraffin-embedded tissue. Arch Pathol Lab Med. 2008;132(12):1929-1935. doi:10.1043/1543-2165132.12.1929

21. Mangan NE, Fallon RE, Smith P, van Rooijen N, McKenzie AN, Fallon PG. Helminth infection protects mice from anaphylaxis via IL-10-producing B cells. J Immunol. 2004;173(10):6346-6356.

22. Pakdel N, Naem S, Rezaei F, Chalehchaleh AA. A survey on helminthic infection in mice (Mus musculus) and rats (Rattus norvegicus and Rattus rattus) in Kermanshah, Iran. Vet Res Forum. 2013;4(2):105-109.

23. Kia E, Shahryary-Rad E, Mohebali M, et al. Endoparasites of rodents and their zoonotic importance in germi, dashte-mogan, ardabil province, iran. Iran J Parasitol. 2010;5(4):15-20.

24. Ceruti R, Sonzogni O, Origgi F, et al. Capillaria hepatica infection in wild brown rats (Rattus norvegicus) from the urban area of Milan, Italy. J Vet Med B Infect Dis Vet Public Health. 2001;48(3):235-240.

25. Reperant LA, Deplazes P. Cluster of Capillaria hepatica infections in non-commensal rodents from the canton of Geneva, Switzerland. Parasitol Res. 2005;96(5):340-342. doi:10.1007/s00436-005-1358-y.

26. Singleton GR, Spratt DM, Barker SC, Hodgson PF. The geographic distribution and host range of Capillaria hepatica (Bancroft) (Nematoda) in Australia. Int J Parasitol. 1991;21(8):945-957.

27. Kia EB, Homayouni MM, Farahnak A, Mohebali M, Shojai S. Study of endoparasites of rodents and their zoonotic importance in ahvaz, south west iran. Iran J Public Health. 2001;30(1-2):49-52.

28. Hasanpor H, Mohebali A, Hajaran H, et al. A Survey On zoonotic helminths of rats in northern parts of Khuzestan province, Iran. J Ilam Univ Med Sci. 2013;21(3):46-52.

29. Nateghpour M, Motevalli-Haghi A, Akbarzadeh K, et al. Endoparasites of wild rodents in southeastern Iran. J Arthropod Borne Dis. 2015;9(1):1-6.

30. Farhang-Azad A. Ecology of Capillaria hepatica (Bancroft 1893) (Nematoda). 1; Dynamics of infection among Norway rat populations of the Baltimore Zoo, Baltimore, Maryland. J Parasitol. 1977;63(1):117-122.

31. Rocha EJ, Basano Sde A, Souza MM, et al. Study of the prevalence of Capillaria hepatica in humans and rodents in an urban area of the city of Porto Velho, Rondonia, Brazil. Rev Inst Med Trop Sao Paulo. 2015;57(1):39-46. doi:10.1590/s0036-46652015000100006. 
32. Elshazly AM, Awad SI, Azab MS, et al. Helminthes of synanthropic rodents (Rodentia: Muridae) from Dakahlia and Menoufia, Egypt. J Egypt Soc Parasitol. 2008;38(3):727740 .

33. Singleton GR, McCallum HI. The potential of Capillaria hepatica to control mouse plagues. Parasitol Today. 1990;6(6):190-193.

34. Singleton GR, Spratt DM. The effects of Capillaria-hepatica (Nematoda) on natality and survival to weaning in Balb/C mice. Aust J Zool. 1986;34(5):677-681.
35. Resendes AR, Amaral AF, Rodrigues A, Almeria S. Prevalence of Calodium hepaticum (syn. Capillaria hepatica) in house mice (Mus musculus) in the Azores archipelago. Vet Parasitol. 2009;160(3-4):340-343. doi:10.1016/j.vetpar.2008.11.001.

36. Farhang-Azad A, Schlitter DA. Capillaria hepatica in small mammals collected from Shoa province, Ethiopia. J Wildl Dis. 1978;14(3):358-361.

37. Scott JP. Agonistic behavior of mice and rats: a review. Am Zool. 1966;6(4):683-701. 